Comunicación y política: ejercicios reflexivos 


\title{
Acción conectiva, acción colectiva y medios digitales: posibilidades para la comunicación política en los tiempos de Internet
}

\author{
Eduardo Villanueva Mansilla \\ (Pontificia Universidad Católica del Perú)
}

Recibido: 31/8/2015

Aprobado: 29/9/2015

\begin{abstract}
Resumen. El presente artículo discute las perspectivas de la comunicación política a través de medios digitales, considerando la capacidad que tienen estos medios de generar dos tipos de acción: conectiva y colectiva, tanto para los movimientos sociales como para los partidos en procesos electorales. La discusión considera la evidencia disponible sobre los éxitos y fracasos asociados a los medios digitales. Se usan tanto los conceptos de la ciencia política como de la comunicación y los estudios de Internet, y plantea la necesidad de analizar desde un marco específico asociado al potencial de los medios para ser aprovechados en contextos determinados.
\end{abstract}

Palabras clave: medios sociales / acción conectiva / medios y engagement / política de contienda / comunicación electoral

\section{Connective Action, Collective Action and Digital Media: Possibilities for Political Communication in Times of the Internet}

Авsтract. This paper discusses the potential for political communication through digital media, taking into account this type of media's ability to produce two types of action: connective action and collective action, both for social movements as well as for political parties during electoral processes. The paper takes into account available evidence about successful and failed experiences in which digital media was used. The analysis uses concepts pertaining both to political science and communication studies as well as Internet studies. However, it raises the need to approach these scenarios through a specific framework that takes into account the media's potential in such particular contexts.

Key words: social media / connective action / engagement and media / contention politics / electoral communication 


\section{Planteamiento}

U na abundancia de opciones aparece ante los ojos de los políticos. De un mundo en donde todo parecía pasar por la televisión y las manifestaciones, hemos pasado a contar con tantos medios como estilos de trabajo. Sin descartar los medios masivos y las viejas formas de comunicación directa, ahora los medios digitales $-\mathrm{y}$ en particular los medios sociales, como Facebook- sugieren que llegar al votante es más fácil y más barato que antes.

Aparece la evidencia, o al menos el reclamo de evidencia, sobre su eficacia para alcanzar movilizaciones singulares y de nuevo cuño en la realidad política contemporánea. De los Indignados europeos a la Primavera Árabe hasta los Pingüinos chilenos, los medios sociales son la ruta para el activismo efectivo y el éxito político. En el Perú, las movilizaciones contra la "ley Pulpín" produjeron entusiasmos interpretados como una señal de renacimiento político de la juventud peruana, y como una esperanza de renovación de los liderazgos de izquierda: jóvenes movilizados digitalmente, organizados de manera horizontal, dispuestos a recrear la política desde su experiencia conectada y conectante (Villanueva, 2015).

Así como en el Perú, donde esas movilizaciones fueron golondrinas de un verano temprano, en el resto del mundo el entusiasmo parece abatirse, y más bien se constata que el gran usuario exitoso de las "redes sociales" es Al-Daesh (ISIS, EI, Estado Islámico), un grupo que ha realizado amplias campañas de difusión y propaganda mediante recursos digitales, tanto para difundir su mensaje como para captar nuevos miembros (Barrett, 2014). Movimientos como Cinque Stelle en Italia o Siriza en Grecia, nacidos al menos en parte en el entusiasmo digital, son ahora simples metáforas movimientistas, incapaces de actuar de manera unificada en la política de su país y superados ampliamente por individuos hábiles como Matteo Renzi o -en el caso griego- Alexis Tsipras.

¿Hay algo en común entre las experiencias exitosas como las de Obama y Al-Daesh y las fallidas como las de Cinque Stelle o la no nacida movilización digital en el Perú? El propósito de este artículo es demostrar que sí; para este fin se hace la comparación entre las características de los distintos medios involucrados y la constatación de la carencia de una base empírica en los juicios favorables sobre el efecto de los medios digitales en la movilización y participación política, sea electoral o movimientista.

Específicamente, las discusiones más repetidas giran alrededor de la transformación de la esfera pública por el uso de los medios digitales, algo sobre lo que se especula desde hace un buen tiempo, con énfasis en el conflicto entre sistemas políticos de masas y experiencias culturales y sociales mucho más complejas, transversales $\mathrm{y}$, a veces, casi privadas (Keane, 1999). Evidentemente, esto afecta de manera directa a 
la política, tanto como contienda en el espacio social como en elecciones para controlar sistemas de gobierno.

El contexto general presenta una serie de propuestas analíticas o conceptuales que proponen que la Internet y los medios digitales cambian profundamente la sociedad, a través de las posibilidades de interconexión $\mathrm{y}$ asociación que ofrecen, creando formas nuevas de poder como el llamado poder en red (Castells, 2011); o a través de las posibilidades de creación colectiva que los efectos de red hacen posible capitalizar socialmente (Benkler, 2006); o quizá permitiendo que la tendencia al 'cosmopolitanismo', propia de sociedades avanzadas, encuentre mecanismos de expansión aún mayores que estarían creando un quinto estamento o una sociedad civil global (Dutton, 2008), o en la llamada sociedad de movimientos (Meyer y Tarrow, 1998). Aparte de las arenas nacionales, la Internet facilita los intercambios transfronterizos y la discusión entre pares más allá de las polities de cada país; el resultado es la evidente presencia de movimientos sociales que recogen o replican actitudes, tácticas o estilos de otros países, en los dos mencionados espacios de acción política.

Sin embargo, es evidente que la relación entre medios digitales y comunicación política continúa demandando investigación. Dos grandes preguntas específicas acerca de este tema aparecen en el terreno: ¿cuál es la capacidad efectiva de movimientos políticos internacionales de influir en un país determinado, con acciones fundamentalmente digitales?, y ¿cuál es el ámbito más efectivo para la comunicación política por medios digitales en contextos electorales? La primera pregunta nos remite a la posibilidad de usar medios digitales para romper la limitación territorial que comparten la comunicación y la política, producto del control que los estados nación han ejercido sobre los ciudadanos y sobre los medios en un territorio estatal determinado: la política es nacional aunque los temas no lo sean, y los medios, de naturaleza nacional, son los que de alguna manera han reforzado esa lectura al estar contenidos en el ámbito nacional.

Pero la segunda pregunta, más que a la naturaleza de la acción política va a la cuestión de las técnicas de comunicación aplicadas a la acción electoral. ¿Es posible ganar una elección usando medios digitales? ¿Es posible trasladar las lecciones de la política electoral de otros países a, digamos, el Perú y tener éxito? No es trivial, dado que muchos consultores, abierta o calladamente, proponen tener la estrategia para el éxito en el uso de herramientas digitales, y que se ha intentado hacerlo en América Latina. Ambas preguntas, además, tienen mucha más relación de lo que parece, dado que el éxito del uso de medios digitales por un movimiento social tendría bases similares a las que un partido político podría tener en una elección: se requiere que la capacidad de difusión de mensajes se traslade a movilización y, finalmente, participación (Downing y Brooten, 2009). 
Tanto el análisis de los movimientos sociales (en sus muchas formas) mejorados y ampliados gracias a la Internet, y la relación entre el uso de Internet y los procesos institucionales, como las elecciones, los partidos políticos y el sistema político en general, presentan una preocupación fundamentalmente similar: de qué manera la Internet puede ser usada para comunicar, movilizar y construir interacción, y finalmente, crear acción colectiva (Lilleker y Vedel, 2013). En el caso de los movimientos, la participación debería resultar en acción colectiva, es decir en actuar como un todo en la contienda política de la calle y la opinión pública, hacia un resultado determinado; en las elecciones, lograr que de elector el ciudadano se convierta en partidario, y haga lo necesario para lograr el triunfo colectivo. Evidentemente, las características propias de los movimientos sociales obligan a un repertorio de investigación distinto al que se requiere para analizar procesos institucionalmente configurados, como las elecciones; hay, sin embargo, lecciones de un ámbito útiles para mejorar el entendimiento del otro.

También para ambos escenarios, las críticas son similares: el "clicktivismo", el reducir la acción política a dar likes o a expresar desagrado en una pantalla, es el único resultado de la acción creada por las conexiones digitales: ni votos ni movilización, solo acción individual delante de una pantalla. Es quizá una crítica excesiva, pero -al mismo tiempo-no hay mucha evidencia de grandes éxitos, sociales o electorales, basados en una postulada acción colectiva digital; y los éxitos suelen ocurrir en contextos precisos, países con sistemas muy consolidados que han requerido inversiones significativas para movilizar a los ciudadanos, a través de campañas muy sofisticadas (Carty, 2011). La movilización y la participación no nace de la ciudadanía o del electorado: son el resultado de acciones muy bien planificadas, tomadas por actores políticos muy profesionales (Williamson, 2010).

Acercarnos, pues, a la resolución de estos debates requiere investigación en el terreno; pero puede servir para plantear la discusión del debate académico sobre la real importancia de los medios digitales. Una revisión del estado del arte puede ser útil para plantearnos las diferencias estructurales entre los medios existentes $\mathrm{y}$, a través de esa ruta, la manera como podríamos resolver las preguntas ya planteadas.

\section{Los términos de la discusión}

Partamos por establecer que la Internet permite una transformación fundamental de la acción de los ciudadanos (Della Porta, 2011; Franklin, 2014), gracias a la existencia de mecanismos de asociación distintos, más diversos y menos concentrados en manos de los actores políticos tradicionales (Jensen, Danziger y Venkatesh, 2007). Entre los posibles ejemplos podemos revisar el de agrupaciones con reivindicaciones sectoriales que logran trasladar su acción al escenario político nacional, co- 
mo el caso de los estudiantes chilenos (Valenzuela, Arriagada y Scherman, 2012); grupos de activismo de derechos femeninos en Indonesia (Molaei, 2014); grupos cosmopolitas antiglobalización (Poell, 2013), como Occupy Wall Street (Jensen y Bang, 2013). También se incluyen estudios que consideran los intentos al parecer exitosos, pero luego agotados rápidamente por falta de repercusión significativa en el espacio social, por variedad de razones: YoSoy132 en México (Treré, 2015); los Indignados de España (Andulza, Cristancho y Sabucedo, 2013); o la finalmente fallida Primavera Árabe, que recibió mucha atención a pesar de los límites rápidamente expuestos de su capacidad transformativa (Howard y Hussain, 2013; Tudoroiu, 2014; Wagner y Gainous, 2013; entre muchos otros).

Dahlgren (2005, p. 153) propone cinco arenas de discusión y comunicación política, brevemente resumidas aquí:

- El e-government, es decir la gestión pública digital. Hay interacción entre ciudadanos y funcionarios públicos, pero se entiende como una extensión de la gestión pública.

- El activismo orientado a la incidencia en políticas, incluyendo el cabildeo y los sindicatos; en los últimos años, los activismos incluyen los movimientos sociales con causas específicas, como la promoción de derechos civiles, de escala internacional.

- La amplia variedad de espacios de deliberación cívica, donde se discute pero no se actúa de manera explícita. Incluye a los medios sociales.

- La discusión prepolítica o parapolítica, donde los temas sociales o culturales asociados a identidades colectivas o intereses comunes se desarrollan discursivamente.

- La prensa, en sus múltiples formas, como espacio de construcción de opinión pública y deliberación de todo tipo; desde la prensa tradicional hasta la digital, en escalas locales, regionales, nacionales e internacionales/globales.

Es evidente que nuestro interés excluye la primera, pero incluye de manera difusa o sobrepuesta las cuatro siguientes, que configuran en un extremo el espacio del activismo más tradicional (incidencia política, por ejemplo) y la prensa en el otro; ahora todas usan de una u otra forma la Internet y los medios digitales. El consenso es que los medios digitales en general y los medios sociales específicamente amplían el repertorio de los movimientos sociales, facilitando la realización de política de contienda, o contention politics, una de las bases de la democratización característica de Europa (y por extensión el resto del mundo bajo polities liberales). La política de enfrentamiento implica necesariamente una lucha de actores que no suele encontrar -sino al final de su desarrollo- expresión electoral, lo que motiva a muchos autores a identificar los desarrollos recientes con el proceso de democratización de larga data, coincidente con el Estado nación 
westfaliano que predomina hoy en el mundo. En palabras de Charles Tilly (2004), uno de los principales teóricos de esta perspectiva,

los repertorios de contienda política (arreglos de performances para proponer reclamos de amplia disponibilidad) se desplazan de interacciones predominantemente parroquiales, particulares y bifurcadas, basadas sobre todo en identidades incrustadas; a interacciones predominantemente cosmopolitas, modulares y autónomas, basadas sobre todo en identidades desprendidas. (p. 8) [Mi traducción]

Esta contienda política coexiste presencial y digitalmente, sin importar el tipo de esfera.

Más allá del exceso de entusiasmo de algunos autores, que entendieron que las redes creadas a través de Internet permitían no solo la ampliación de esferas comunicativas, sino una transformación social efectiva y radical (Castells, 2012, es el ejemplo más desbordado de ese optimismo), hay una variedad de trabajos que muestran tanto el potencial como las limitaciones de los medios digitales (Chadwick, 2013), y -particularmente- plantean un debate que originalmente aparece en las reflexiones sobre el impacto de los medios masivos en la política en democracias consolidadas, planteado por Antonio Mellucci (1996, 1999) y recogido por muchos otros autores: ¿es posible realmente alcanzar acción colectiva, o apenas se logra acción "conectiva" mediante la actividad mediática? Es decir, reemplazado el espacio público tradicional de las masas movilizadas de inicios del siglo $X X$ por la sociedad mediada, ¿acaso la acción colectiva no será posible, y solo se puede postular movilizaciones limitadas alimentadas desde la conectividad mediada?

Las identidades colectivas que llevan a la acción colectiva, y que a su vez crearon los partidos de masas del siglo $\mathrm{XX}$, estarían siendo reemplazadas por

actuar públicamente o contribuir a un bien común como un acto de expresión y reconocimiento personal, o autovalidación alcanzada por el compartir de ideas y acciones en relaciones basadas en confianza ${ }^{2}$ (Bennett y Segerberg, 2012, p. 752). [Mi traducción]

Esta reflexión es crítica y sirve especialmente para discutir los movimientos sociales digitales, tanto en su forma intensa o activa, llamada hacktivismo por Jordan y Taylor (2004), como lo que muchas veces se califica -algo despectivamente- como "click-

1 "Repertoires of political contention (arrays of widely available claim-making performances) shift from predominantly parochial, particular, and bifurcated interactions based largely on embedded identities to predominantly cosmopolitan, modular, and autonomous interactions based largely on detached identities".

2 "Public action or contributing to a common good [as] an act of personal expression and recognition or self-validation achieved by sharing ideas and actions in trusted relationships". 
tivismo". A su vez, estudios como el de Ponder y Haridakis (2015) permiten especular que el uso de medios sociales suele producir mucho más interés en reforzar las posiciones que ya se tienen, discutiendo con miembros de grupos de opinión que coinciden con el interlocutor; mientras que en el caso de los medios masivos, lo que suele ocurrir es mayor intercambio con el out-group; esta idea es válida tanto para los participantes de movimientos sociales como para los ciudadanos en situaciones electorales o preelectorales convencionales; aunque Lee, Choi, Kim y Kim (2014) apuntarían en la dirección opuesta.

Respecto al ámbito electoral, en que se privilegia el resultado específico en vez de la construcción de un movimiento, la situación es distinta. Hay evidentes éxitos, así como fracasos más o menos claros: el primer caso fue el del estadounidense Howard Dean, candidato en las primarias demócratas de 2004, que movilizó recursos y personas gracias al uso de plataformas digitales; su fracaso se atribuye más a sus limitaciones como candidato que propiamente a la estrategia (Gil de Zúñiga, Veenstra, Vraga y Shah, 2010). Barack Obama, tanto en 2008 como en 2012, hizo un uso fino y constante de los recursos digitales, y es posible atribuir al menos una parte importante de su victoria a dos factores: identificación de votantes potenciales y movilización de voluntarios para captarlos, algo difícil en elecciones de voto facultativo y que además se definen regionalmente, por colegio electoral, y no por una mayoría absoluta nacional (Bimber, 2014; Towner, 2012). En cambio, la francesa Segolene Royal no logró conectar su propuesta de movilización electoral con un voto favorable en 2009 (Gibson, Römmele y Williamson, 2014); en el Reino Unido, ni en 2010 ni en 2015 parece que la influencia de la Internet haya sido decisiva en las elecciones británicas, con la atingencia de que no hay todavía evaluaciones académicas de sus más recientes comicios (Williamson, 2010). Finalmente, dos casos especiales: la movilización electoral del movimiento Cinque Stelle, en Italia, que convirtió a una coalición muy variada, dirigida por un comediante, en una fuerza electoral (aunque esta no ha logrado tener peso político por deficiencias en su accionar parlamentario) (Cammaerts, Doudaki, Askanius, De Cleen, González y Trivundza, 2015); y el fracaso de la única campaña claramente digital en América Latina, el partido Oxígeno Verde de Antanas Mockus, que fracasó a pesar de las expectativas que creó en Colombia para las elecciones presidenciales de 2011 (Rincón, 2011). La tendencia es a establecer bajo qué circunstancias, tanto institucionales como comunicacionales, es posible lograr éxito mediante recursos digitales: colaborar en una victoria electoral, lograda gracias a la difusión, participación y movilización de personas y recursos, usando medios digitales como parte integral de la estrategia (Breindl, 2013; Copeland y Römmele, 2014; entre otros). 
El consenso indica un proceso complejo de desarrollo de servicios y experiencias que coincide con el despliegue de una multiplicidad de medios digitales, creados con fines comerciales en su mayoría, y que no tenían interés u orientación política. El ejemplo inicial ya mencionado, la experiencia de Howard Dean, nació en un sitio llamado meetup, pensado para facilitar encuentros de pareja, pero que fue explotado para encuentros entre partidarios del candidato. Luego fue el uso de distintas plataformas, como los blogs o los sitios de conversación (chat rooms y similares). Sin embargo, desde 2010 predomina el uso de Facebook y Twitter, dos sitios funcionalmente muy distintos pero que son calificados como medios sociales, es decir sitios dedicados a facilitar y promover la creación de contenidos por sus usuarios (Boyd y Ellison, 2010). Son estos medios sociales los que resultan fundamentales para entender tanto los movimientos sociales digitales como los procesos políticos que aprovechan esta "vida digital" (Treré y Carretero, 2013; Trottier y Fuchs, 2015), ampliando el repertorio de los grupos políticos y eventualmente cuestionando las bases de los regímenes políticos tradicionales.

En este panorama aparece una divergencia clara: lo que hace interesante a los medios digitales en contexto de contienda social o elecciones no es la capacidad de difusión sino la conexión entre los ciudadanos, esa "acción conectiva" que sería el resultado base de la utilización de lo digital para la política, con la acción colectiva como resultado complejo, multivariable $\mathrm{y}$ no necesario, de lo anterior. Es decir, sea para movilizar socialmente o para lograr participación intensa en elecciones, los medios digitales conectan a personas y les dan un espacio, pero no está claro si este espacio solo existe en el medio digital, o si es posible pensar que luego de creado en lo digital podría pasar a ocurrir también en el ámbito presencial (Vissers y Stolle, 2013).

Pero no parece haber evidencia de que esas personas conectadas hayan sido convencidas. Todos los casos mencionados son particulares porque traen resultados interesantes que luego se enfrentan con limitaciones institucionales serias, como pasó en los países árabes o con los Indignados; o porque el resultado alcanzado, la elección de un candidato como Obama, no influye en el sistema político para que permita el sostenimiento del movimiento, y -por lo tanto- la transformación de la agenda política. Es decir, la conexión moviliza para un logro específico y luego parece agotarse en sí misma.

Para una comprensión más cuidadosa de esta situación, deben analizarse dos dimensiones por separado. Por un lado, han de considerarse las condiciones institucionales para el desarrollo de la contienda política o electoral; pero, por otro lado, los medios usados para que la contienda tenga lugar crean condiciones de acción específicas. Por ello, en este artículo se revisará la na- 
turaleza de los medios involucrados, es decir la manera como los medios digitales configuran las posibilidades de acción, conexión y participación. Evidentemente, hay diferencias entre los viejos medios masivos y los nuevos medios digitales. Esas diferencias sirven para esbozar explicaciones de las limitaciones del activismo digital.

\section{Los medios digitales y su recurso al engagement}

Los llamados medios sociales incluyen aquellos que son alimentados fundamentalmente por sus usuarios, como los servicios de red social (Facebook es el más difundido, pero ciertamente no el único); los de micromensajería/ microblogueo con redifusión, como Twitter o Sina Weibo; o aquellos que usan de manera intensiva el contenido creado por sus usuarios para aumentar la circulación de sus contenidos, como YouTube.

En todos los casos, la importancia del contenido social reside en la manera como cada consumidor/usuario se relaciona con otras personas a través del medio, creando un tejido de intercambios que facilita un mayor flujo de mensajes, pero también de sentimientos, opiniones y desagrados. Los contenidos de mayor interés producen un aumento del tráfico, por lo general acompañado de manifestaciones de aprobación diversas. La experiencia no se reduce a recibir contenidos y comentarlos "fuera de línea", sino que el intercambio en línea se expande al incluir las diversas dimensiones de la socialidad expresadas en los medios digitales.

A esta forma de relación con los medios se le ha llamado engagement ${ }^{3}$. A diferencia de su definición política, un sentido de involucramiento en los asuntos de la vida política (Dahlgren y Alvares, 2013), engagement en relación a la experiencia mediática digital refiere a la manera como los medios sociales generan reacciones dentro del medio por parte de los usuarios: las manifestaciones de estas reacciones, de estas tomas de posición, como el "me gusta" y el "compartir" de Facebook (FB), o el "retuiteo/RT" de Twitter, implican que la expresión de gusto o aprobación se manifiesta en acciones que promueven el contenido específico más allá de la pantalla del usuario y en dirección a aquellos que conforman la red de relaciones que se ha construido a través del uso: los "amigos" o contactos en FB, o los seguidores en Twitter. Aprobar con un like un mensaje en FB significa coincidencia o gusto por el mensaje; compartirlo equivale a querer ampliar su alcance, de manera similar con el retuiteo. Más que el número bruto de amigos o contactos que

3 Al no haber en el idioma español una traducción aceptada de este término, se le seguirá usando en inglés. 
una persona puede tener, es el grado de replicación y redifusión en la red particular de contactos lo que muestra el impacto que determinado contenido tiene; un usuario que logra continuamente esa replicación y redifusión obtiene mayor engagement de parte de su red de contactos.

Ese engagement es una característica inherente a este tipo de medios, que privilegia la conexión a partir de intereses y experiencias comunes; esos intereses no implican más acción que el compartir y quizá la interlocución, en forma de comentarios y respuestas. No requieren que el tejido de conexiones salga del espacio digital en donde ocurre, sino que más bien ese tejido medra en la facilidad de su uso y la relativa irresponsabilidad de las interacciones que facilita. El engagement tiene una dimensión emocional y de satisfacción personal, que en la política debería incluir el logro no solo individual sino también el contexto colectivo. Es mucho más fácil cuando se trata de consumo mediático, en el amplio sentido; la satisfacción es el hecho de compartir la experiencia. En política, en cambio, el logro debería estar asociado a las posibilidades de un resultado claro, de un cambio en la gestión de las cosas.

Los medios que se centran en generar engagement han sido llamados por Jenkins, Ford y Green (2012) spreadable media: medios que se pueden expandir sobre las superficies sociales como si se untaran; no se difunden a partir de centros específicos, sino que lentamente penetran la sociedad, pero también pueden ser usados para desparramar su influencia en superficies cada vez más amplias. El grueso del análisis gira sobre el consumo y sobre las llamadas culturas de fans y de convergencia que han llamado la atención en los últimos años; esa base analítica sirve para acercarse a los medios en general: estamos ante un potencial de reutilización, reciclaje y de apropiación personal de los mensajes a través de estos spreadable media; la política también puede buscarlo.

El poder de un medio social, entonces, resulta de su potencial para facilitar que sus usuarios se apropien del contenido, lo expandan y unten sobre otros espacios sociales; esto remite al engagement, como un compromiso asumido personalmente de encontrar individuos de similares intereses y gustos, a los cuales el contenido les será particularmente grato. Si bien esta reflexión se inicia en el ámbito del consumo mediático en general, la aplicación de ese concepto a la política es clara: un medio social permite la intensificación de los intercambios y la afirmación de convicciones entre los que ya son partidarios; pero un entorno de intercambios muy intenso es poco atractivo para aquel que puede tener dudas, no está convencido o simplemente no tiene el mismo interés que aquellos ya dedicados a difundir el mensaje.

Podemos notar entonces que los medios sociales, al ser usados en política, servirán sobre todo para motivar a que quienes comparten ciertas ideas se en- 
cuentren entre sí, logrando que personas que piensan similarmente puedan expresarse y actuar del mismo modo en el espacio virtual; por ello, podrán solidificar sus convicciones y fortalecer sus puntos de vista. Pero el caso es que no es necesariamente la mejor forma de convencer a las personas que no se han formado una opinión sobre determinados asuntos; es más, puede ser que sea contraproducente exponer a las personas no convencidas a estos medios, porque la participación de los convencidos puede crear un entorno altamente partidarizado y poco atractivo para el que está en proceso de formar una opinión.

Esto exige preguntarse necesariamente por la utilidad de este tipo específico de engagement para fomentar la participación política efectiva, entendida esta como llevar las intenciones a la acción colectiva desde los ejercicios de conexión. ¿Cuán importante es para el engagement el contexto sociopolítico? ¿Cuán definidor es contar con recursos para el uso de los medios digitales? Todo indicaría que los jóvenes, que no solo tienen más hábito de uso de estos medios sino también más tiempo disponible, son los que más participan en el engagement político; y que a pesar de la creencia acerca del poder igualador de la Internet, es también importante conocer el valor de los recursos materiales y educacionales en la identificación del uso de los medios con fines políticos (Anduiza, Gallego y Cantijoch, 2010; Koc-Michalska, Gibson y Vedel, 2014).
Es precisamente en las sociedades donde el acceso a los recursos es significativo, sobre todo a los digitales, y donde determinadas situaciones políticas que superan al sistema existente permiten el surgimiento de movilizaciones distintas, que los medios digitales resultan relevantes para entender la naturaleza de esos movimientos y el eventual éxito y continuidad de cada uno. El caso de los Indignados españoles es el mejor ejemplo: gracias a la conectividad a través de medios digitales, grupos altamente dispersos pero con convicciones similares logran coligarse en actividades masivas en el espacio público, con impactos directos, inmediatos, pero sin lograr trascender la actividad movilizadora hacia un espacio electoral o formal (Anduiza, Cristancho y Sabucedo, 2013; Micó y Casero-Ripollés, 2013). Estas nociones se pueden generalizar, a partir del análisis realizado tanto por Ang, Dinar y Lucas (2014) como por Theocharis, Lowe, van Deth, y Albacete (s. f.) a la mayoría de casos de movilización, y se puede añadir que la capacidad de conexión generada en los medios digitales actúa como vía de catalización; así como ciertas situaciones se convierten en catalizadores sociales de malestares contenidos o latentes que no logran expresión a través del sistema político formal, sea por deficiencias locales (lo que podría postularse sobre lo que ocurrió en 2014 y 2015 en Lima con la "ley Pulpín") o limitaciones estructurales producto del autoritarismo (como en el caso de la Primavera Árabe). 
Al mismo tiempo, estos medios permiten un tipo de seguimiento mucho más intenso y detallado que los viejos medios masivos. Es posible rastrear la difusión de mensajes en medios sociales en tiempo real, así como hacer mapas o gráficos de las relaciones que se crean a través de estos medios por parte de actores políticos específicos. Esto permite identificar patrones de largo plazo que han servido para estimar el verdadero impacto en la construcción de movimientos sociales, y también por parte de actores políticos formales, en ocasiones específicas, como puede verse en Hooghe et al. (2010), González-Bailón et al. (2011), o Thorson et al. (2013) para movimientos sociales; y para partidos políticos, en Jackson y Lilleker (2009), Earl y Kimport (2014), y Xenos, Vromen y Loader (2014), entre otros.

Son precisamente los actores políticos más profesionales los que logran hacer mejor uso de las posibilidades del engagement, no solo al producir contenido sino al alimentar a los grupos de interesados o partidarios, identificados por estas herramientas de seguimiento, a los que se les facilita y promueve como usuarios de contenido.

Wallsten (2010) explica cómo fue la campaña de Obama, a la que logró convertir en viral a través de constantes actos de promoción y motivación con individuos destacados en las redes de partidarios; en este caso el video de campaña Yes We Can fue interpretado originalmente como un caso de "espontaneidad" de parte del público. En otro contexto, Porten-Cheé (2013) explica cuánto influye en el uso de los sitios web de los partidos políticos alemanes la promoción muy focalizada que realizan los especialistas en comunicación de esas organizaciones.

Así, retomando el argumento inicial, lo que tenemos como resultado del uso de medios digitales en política es acción conectiva, antes que colectiva: no ocurre el tipo de acción asociada con altos niveles de recursos organizacionales y la formación de identidades colectivas, sino el intercambio personalizado de contenidos a través de redes mediáticas (Bennett y Segerberg, 2012).

Esto no cambia la dinámica fundamental de la acción realizada; es más, la limita profundamente, pues la hace dependiente del entusiasmo que específicamente se pueda provocar en cada caso, de acuerdo con las estrategias de los líderes, como se ha explicado en lo relacionado con las campañas de Barack Obama.

¿Qué se puede concluir a partir de la predominancia de la acción conectiva? Hay lecciones concretas para la comunicación política. Aunque lo más interesante para el académico, pero sin duda aún más para el profesional, es la cantidad de preguntas pendientes.

\section{¿Es posible ofrecer algo concreto? El peso de las condiciones institucionales}

La comunicación política nació en un territorio con fronteras más definidas, 
donde los medios eran pocos y las esferas no se superponían; muchas de las prácticas fueron, además, resultado de contiendas políticas, electorales o no, en sociedades más homogéneas, con partidos de masas y de apelación general. En la actualidad, tenemos cada vez mayor heterogeneidad cultural y social, en un entorno mediático mucho más grande, disperso y con medios que se dirigen a públicos de tamaños muy distintos; a su vez, los ciudadanos/consumidores participan en públicos muy diversos y son medidos como audiencias de muy distintas experiencias. A esto hay que añadir el aumento de los mediadores políticos, desde periodistas hasta "opinólogos" que en Facebook logran seguidores que no son representativos para una escala de masas, pero que son importantes para sus pequeñas y motivadas audiencias.

El resultado es una serie de cámaras de resonancia donde cada quien encuentra su propia narrativa de lo político; acompañada de cinismo y falta de engagement por parte de los ciudadanos, frente a convencidos involucrados en la defensa de posiciones precisas, casi cerradas a la discusión (Blumler y Kavanagh, 1999).

Sea para movimientos sociales o para los partidos que participan en elecciones, la meta es alcanzar la acción colectiva, que es lo que debería llevar al logro final. Pero esto no niega que parte del desafío digital sea definir qué es acción colectiva, y si es deseable. Asumiendo que para un movimiento social es necesaria la sostenibilidad luego del entusiasmo inicial, y que para un partido político es requisito una militancia que sirva para alcanzar la meta de mayores votos, emergen dos cuestiones claras:

1. El logro final requiere conocer las particularidades del terreno en que se desarrollará la acción conectiva. En un sistema electoral, las metas deben ser lograr participación concreta y efectiva, por ejemplo en la forma que la campaña de Obama alcanzó: voluntarios para motivar a los votantes menos comprometidos a participar en la elección; en un país como el Perú, donde el voto es obligatorio, el objetivo parece ser más lograr un voto con convicción, que provoque en el votante realizar una difusión y movilización entre su red de contactos, antes del acto electoral mismo. Para los movimientos sociales, la claridad de las metas servirá para definir el objetivo, pero la pregunta por la continuidad no parece tener una resolución clara.

2. El tema que salta inmediatamente es el de los recursos. No está de más recoger una observación de Pierson (2000, p. 258), quien sostiene que la acción colectiva carece de relación lineal entre esfuerzo y efecto. Dicho de otra forma: la acción colectiva resulta de un efecto multiplicador, cuando los esfuerzos de motivación, organización y movilización consiguen que muchas personas emprendan la tarea escogida y se comprometan. Los límites de la acción colectiva en entornos como la Internet yacen en la naturaleza de las interacciones, en donde cada individuo es capaz 
de decir o hacer cosas de inmediato beneficio personal, pero que no lo comprometen a fortalecer el conjunto.

Cualquiera de estas redes de contenidos está compuesta por al menos dos grandes grupos: los proveedores y los consumidores; y mientras que los proveedores tienen sus propias motivaciones para continuar realizando su labor, los consumidores solo buscan la satisfacción personal (Dreyfuss, 2000). A diferencia de los entornos sociales "reales", aquí es fácil retraerse en el anonimato y evadir la responsabilidad de las acciones individuales. La tendencia natural de las personas al free-riding (Lemley, 2005), es decir a consumir sin aportar más allá de lo mínimo indispensable, resulta siendo reforzada por redes que facilitan la circulación de bienes pero que no requieren compromiso $o$ actividades que refuercen las estructuras sociales que conforman las redes.

Esto no es distinto en la acción conectiva. Satisfecha la necesidad de decir algo, o de encontrar personas que piensen igual, no hay razón para trascender el intercambio si es que no se propone algo más concreto, y si no se plantea eso en forma de logro compartido, no solo a partir de los intereses del candidato o de los líderes sino del participante en la interlocución: el ciudadano busca un espacio para sentirse relevante, para involucrarse en el sentido ya citado de Dahlgren y Alvares, lo que implica una dimensión subjetiva que no puede reducirse a líneas en una lista de talking points: tiene que incorporar el sentido de relevancia perdido en la política contemporánea. Ese engagement político no es difícil de lograr a partir del engagement mediático al que apela Jenkins; lo difícil es el engagement emocional cuando la satisfacción no es inmediata.

Entonces, la secuencia parece clara: más allá de entusiasmos aparentes dentro de la red de partidarios ya movilizados y participantes, una campaña política en medios digitales requerirá de una intensa dedicación para sostener tanto la abundancia de contenidos nuevos y renovados que mantengan el interés de la audiencia, como movimientos tácticos claros para lograr que el engagement no solo se sostenga sino que se generalice más allá del grupo inmediatamente motivado. Finalmente, la motivación tras el engagement -es decir, el objetivo que se busca alcanzar colectivamente- debe estar definida con claridad, no en términos de resultado de interés para el que convoca, es decir para el candidato o el líder, sino para aquellos que son convocados.

La ejecución de esa secuencia dependerá de las condiciones institucionales, tanto como de la coyuntura específica. La experiencia más exitosa, las campañas de Obama, fijaron sus logros a partir de las características, las debilidades y las oportunidades del sistema político, por ejemplo al movilizar votantes como voluntarios y darle ownership, propiedad sobre una parte del logro. Las que se iniciaron bien pero no pudieron continuar tras 
el logro inmediato, como la revolución de enero en Egipto, fueron el resultado de aluviones de apoyo que luego no encontraron una ruta de supervivencia. Las que se agotaron en sí mismas, como los Indignados u Occupy Wall Street, no tenían el objetivo claro que resultaría de la movilización. La acción colectiva requiere que trascienda la comunicación y convertir la voluntad de conexión en participación; la movilización digital sin el componente final termina en frustración.

No es una novedad. Si la comunicación política nace de las realidades políticas del siglo $\mathrm{XX}$, debe ahora transformarse para ser útil en las realidades confusas y variadas del siglo XXI. En particular, los medios digitales pueden ser los mismos en muchas realidades, como Facebook y su predominio global, pero responden a condiciones de acción locales de una manera mucho más intensa de lo que a veces parece reconocerse. Parafraseando a Bakardjieva (2015, p. 989), si bien la materialidad formativa de las tecnologías de comunicación es importante, no se debe caer en la tentación de derivar la lógica de la acción política de la estructura del medio. Esta observación es ciertamente válida para todo caso de política mediatizada, y asumirlo -siguiendo con Bakardjieva- implica reconocer el laborioso examen de las minucias de los diversos contextos, las situaciones políticas concretas, la configuración de los actores sociales y el contenido de las demandas y luchas concretas.

\section{Referencias}

Anduiza, E., Cristancho, C., y Sabucedo, J. M. (2013). Mobilization through online social networks: the political protest of the indignados in Spain. Information, Communication $\mathcal{E}$ Society, 17(6), 750-764. DOI: 10.1080/1369118x.2013.808360

Anduiza, E., Gallego, A., y Cantijoch, M. (2010). Online political participation in Spain: the impact of traditional and Internet resources. Journal of Information Technology E Politics, 7(4),356368. DOI: 10.1080/19331681003791891

Ang, A. U., Dinar, S., y Lucas, R. E. (2014). Protests by the young and digitally restless: the means, motives, and opportunities of antigovernment demonstrations.Informa tion, Communication \& Society, 17(10), 1228-1249. DOI: 10.1080 /1369118x.2014.918635

Bakardjieva, M. (2015). Do clouds have politics? Collective actors in social media land. Information, Communication \& Society, 18(8), 1-8. DOI: 10.1080/1369118x.2015.1043320

Barrett, R. (2014). The Islamic State. Nueva York: Soufan Group.

Benkler, Y. (2006). The wealth of networks: how social production transforms markets and freedom. New Haven: Yale University Press.

Bennett, L. W., y Segerberg, A. (2012). The logic of connective action. Information, Communication $\mathcal{E}$ Society, 15(5), 739-768. DOI: 10.1080/1369118x.2012.670661 
Bimber, B. (2014). Digital media in the Obama campaigns of 2008 and 2012: adaptation to the personalized political communication environment. Journal of Information Technology \& Politics, 11(2), 130-150. DOI: 10.1080/19331681.2014.895691

Blumler, J. G., y Kavanagh, D. (1999). The third age of political communication: influences and features. Political Communication, 16(3), 209230. DOI: 10.1080/105846099198596.

Boyd, D., y Ellison, N. (2010). Social network sites: definition, history, and scholarship. IEEE Engineering Management Review, 38(3), 16-31. DOI: 10.1109/emr.2010.5559139

Breindl, Y. (2013). Assessing success in Internet campaigning. Information, Communication \& Society, 16(9), 14191440.DOI:10.1080/1369118x.2012.707673

Cammaerts, B., Doudaki, V., Askanius, T., De Cleen, B., González, P., y Trivundža, I. T. (2015). Pirates on the liquid shores of liberal democracy: movement frames of European pirate parties. Javnost-The Public. DOI: 10.1080/13183222.2015.1017264

Carty, V. (2011). Wired and mobilized: social movements, new technology and electoral politics. Abingdon: Routledge.

Castells, M. (2011). A network theory of power. International Journal of Communication, 5, 773-787. Recuperado de http://ijoc.org/index.php/ ijoc/article/view/1136

Castells, M. (2012). Networks of outrage and hope. Cambridge: Polity.
Chadwick, A. (2013). The hybrid media system: politics and power. Oxford: Oxford University Press.

Copeland, L., y Römmele, A. (2014). Beyond the base? Political parties, citizen activists, and digital media use in the 2009 German Federal election campaign. Journal of Information Technology $\mathcal{E}$ Politics, 11(2), 169-185. DOI: 10.1080/1933 1681.2014.902783

Dahlgren, P. (2005). The Internet, public spheres, and political communication: dispersion and deliberation. Political Communication, 22(2), 147-162. DOI: 10.1080/10584600590933160

Dahlgren, P., y Alvares, C. (2013). Political participation in an age of mediatisation. Javnost-The Public, 20(2), 47-65. DOI: 10.1080/13183222.2013.11009114

Della Porta, D. (2011). Communication in movement: social movements as agents of participatory democracy. Information, Communication $\mathcal{E}$ Society, 14(6), 800-819. DOI: 10.1080/1369118x.2011.560954

Downing, J. D. H., y Brooten, L. (2009). ICTs and political movements. En Ch. Avgerou, R. Mansell, D. Quah y R. Silverstone (Eds.), The Oxford handbook of information and communication technologies (537-544). Oxford: The University Press.

Dreyfuss, H. (2000). Anonimato y compromiso en la época actual: Sören Kierkegaard y el Internet. Areté, Revista de Filosofía, 12(1). Recuperado de http://revistas.pucp.edu.pe/index. php/arete/article/view/5601 
Dutton, W. (2008). The fifth estate, democratic social accountability through the emerging network of networks. Oxford Internet Institute. Recuperado de http://papers.ssrn.com/sol3/papers. cfm?abstract_id=1167502

Earl, J., y Kimport, K. (2014). Digitally enabled social change: activism in the Internet age. Cambridge, MA: MIT Press (MA).

Franklin, M. I. (2014). Digital dilemmas: power, pesistance, and the Internet. Oxford: Oxford University Press.

Gibson, R., Römmele, A., y Williamson, A. (2014). Chasing the digital wave: international perspectives on the growth of online campaigning. Journal of Information Technology $\mathcal{E}$ Politics, 11(2), 123-129. DOI: 10.1080/19331681.2014.903064

Gil de Zúñiga, H., Veenstra, A., Vraga, E., y Shah, D. (2010). Digital democracy: reimagining pathways to political participation. Journal of Information Technology \& Politics, 7(1), 36-51. DOI: $10.1080 / 19331680903316742$

González-Bailón, S., Borge-Holthoefer, J., Rivero, A., y Moreno, Y. (2011). The dynamics of protest recruitment through an online network. Scientific Reports. DOI: 10.1038/srep00197

Hooghe, M., Vissers, S., Stolle, D., y Mahéo, V.-A. (2010). The potential of Internet mobilization: an experimental study on the effect of Internet and face-toface mobilization efforts. Political
Communication, 27(4), 406-431. DOI: 10.1080/10584609.2010.516799

Howard, P. N., y Hussain, M. M. (2013). Democracy's Fourth Wave?: Digital Media and the Arab Spring. Oxford: Oxford University Press.

Jackson, N. A., y Lilleker, D. G. (2009). Building an architecture of participation? Political parties and web 2.0 in Britain. Journal of Information Technology \& Politics, 6(3-4), 232-250. DOI: $10.1080 / 19331680903028438$

Jenkins, H., Ford, S., y Green, J. (2012). Spreadable media: creating value and meaning in a networked culture. New York: New York University Press.

Jensen, M. J., y Bang, H. P. (2013). Occupy Wall Street: a new political form of movement and community? Journal of Information Technology $\mathcal{E}$ Politics, 10(4), 444-461. DOI: 10.1080/19331681.2013.803948

Jensen, M. J., Danziger, J. N., y Venkatesh, A. (2007). Civil society and cyber society: the role of the Internet in community associations and democratic politics. The Information Society, 23(1), 39-50. DOI: 10.1080/01972240601057528

Jordan, T., y Taylor, P. A. (2004). Hacktivism and cyberwars. New York, NY: Routledge.

Keane, J. (1999). Lo público en la era de la abundancia comunicativa. En M. Giusti y M. I. Merino. Ciudadanos en la sociedad de la información (pp. 2340). Lima: Pontificia Universidad Católica del Perú, Fondo Editorial. 
Koc-Michalska, K., Gibson, R., y Vedel, T. (2014). Online campaigning in France, 2007-2012: political actors and citizens in the aftermath of the web.2.0 evolution. Journal of Information Technology $\mathcal{E}$ Politics, 11(2), 220-244. DOI: 10.1080/ 19331681.2014.903217

Lee, J. K., Choi, J., Kim, C., \& Kim, Y. (2014). Social media, network heterogeneity, and opinion polarization. Journal of Communication, 64(4), 702-722. DOI: 10.1111/jcom.12077

Lemley, M. A. (2005). Property, intellectual property, and free riding. SSRN Electronic Journal. DOI: 10.2139/ssrn.582602

Lilleker, D. G., y Vedel, T. (2013). The Internet in campaigns and elections. En W. H. Dutton (Ed.), The Oxford handbook of Internet studies (pp. 401-420). Oxford, England: Oxford University Press.

Margetts, H. (2013). The Internet and democracy. En W. H. Dutton (Ed.), Oxford handbook of Internet studies (pp. 421-437). DOI: 10.1093/oxfordhb/9780199589074.013.0020

Melucci, A. (1996). Challenging codes: collective action in the information age. Cambridge: Cambridge University Press.

Melucci, A. (1999). Acción colectiva, vida cotidiana y democracia. México, D. F.: El Colegio de México, Centro de Estudios Sociológicos.

Meyer, D. S., y Tarrow, S. (1998). A movement society: contentious poli- tics for a new century. En D. S. Meyer y S. Tarrow (Eds.), The social movement society: contentious politics for a new century (1-28). Oxford: Rowman and Littlefield.

Micó, J.-L., y Casero-Ripollés, A. (2013). Political activism online: organization and media relations in the case of $15 \mathrm{M}$ in Spain. Information, Communication \& Society, 17(7), 858-871.DOI: 10.1080/1369118x. 2013.830634

Molaei, H. (2014). Discursive opportunity structure and the contribution of social media to the success of social movements in Indonesia. Information, Communication \& Society, 18(1), 94-108. DOI: 10.1080/1369118x.2014.934388

Pierson, P. (2000). Increasing returns, path dependence, and the study of politics'. The American Political Science Review, 94(2), p. 251. DOI: 10.2307/2586011

Poell, T. (2013). Social media and the transformation of activist communication: exploring the social media ecology of the 2010 Toronto G20 protests. Information, Communication $\mathcal{E}$ Society, 17(6), 716-731. DOI: 10.1080/1369118x.2013.812674

Ponder, J. D., y Haridakis, P. (2015). Selectively social politics: the differing roles of media use on political discussion. Mass Communication and Society, 18(3), 281-302. DOI: 10.1080/15205436.2014.940977

Porten-Cheé, P. (2013). The use of party web sites and effects on voting: the 
case of the European parliamentary elections in Germany in 2009. Journal of Information Technology $\mathcal{E}$ Politics, 10(3), 310-325. DOI: 10.1080/19331681.2013.803946

Rincón, O. (2011). Mucho ciberactivismo... pocos votos: Antanas Mockus y el Partido Verde colombiano. Nueva Sociedad, 235, 74-89.

Theocharis, Y., Lowe, W., van Deth, J. W., y Albacete, G. G. (s. f.). Using Twitter to mobilise protest action: transnational online mobilisation patterns and action repertoires in the Occupy Wall Street, Indignados and Aganaktismenoi movements. SSRN Electronic Journal. DOI: 10.2139/ssrn.2221824

Thorson, K., Driscoll, K., Ekdale, B., Edgerly, S., Thompson, L. G., Schrock, A., Swartz, L., Vraga, E. K., y Wells, C. (2013). YouTube, Twitter and the Occupy Movement. Information, Communication \& Society, 16(3), 421-451. DOI: 10.1080/ 1369118x.2012.756051

Tilly, C. (2004). Contention and democracy in Europe, 1625-2000. Cambridge, MA: Cambridge University Press.

Tilly, C. (2007). Regimes and repertoires. Chicago, IL: University of Chicago Press.

Towner, T. L. (2012). Campaigns and elections in a Web 2.0 world: uses, effects and implications for democracy. En Ch. G. Reddick y S. K. Aikins (Eds.), Web 2.0 technologies and democratic governance: political, policy and management implications (185-199). Nueva York: Springer.
Treré, E. (2015). Reclaiming, proclaiming, and maintaining collective identity in the \#YoSoy132 movement in Mexico: an examination of digital frontstage and backstage activism through social media and instant messaging platforms. Information, Communication $\mathcal{E}$ Society, 18(8), 1-15. DOI: 10.1080/ 1369118x.2015.1043744

Treré, E., y Carretero, A. (2013). De mitos y sublimes digitales: movimientos sociales y tecnologías de la comunicación desde una perspectiva histórica. Revista de Estudios para el Desarrollo Social de la Comunicación, 8, 27-47. DOI: 10.15213/redes.n8.p27

Trottier, D., y Fuchs, C. (Eds.). (2015). Social media, politics and the state: protests, revolutions, riots, crime and policing in the age of Facebook, Twitter and YouTube. United Kingdom: Routledge.

Tudoroiu, T. (2014). Social media and revolutionary waves: the case of the Arab Spring. New Political Science, 36(3), 346-365. DOI: 10.1080/ 07393148.2014.913841

Valenzuela, S., Arriagada, A., y Scherman, A. (2012). The social media basis of youth protest beha vior: the case of Chile. Journal of Communication, 62(2), 299-314. DOI: 10.1111/j.1460-2466.2012.01635.x

Villanueva Mansilla, E. (2015). De pulpines e indignados: una opinión sobre el fin sin inicio del poder en red en el Perú. Ideele, 249. Recuperado de http://revistaideele. com/ideele/content/de-pulpines-eindignados-una-opini $\%$ C3\%B3n- 
sobre-el-fin-sin-inicio-del-poderen-red-en-el-per $\%$ C3\%BA.

Vissers, S., yStolle, D. (2013). The Internet and new modes of political participation: online versus offline participation. Information, Communication $\mathcal{E}$ Society, 17(8), 937-955. DOI: 10.1080/1369118x.2013.867356

Wagner, K. M., y Gainous, J. (2013). Digital uprising: the Internet revolution in the Middle East. Journal of Information Technology $\mathcal{E}$ Politics 10(2), 261-275. DOI: 10.1080/19331681.2013.778802

Wallsten, K. (2010). "Yes We Can": how online viewership, blog discussion, campaign statements, and mainstream media coverage produced a viral video phenomenon. Journal of Information Technology $\mathcal{E}$ Politics, 7(2-3), 163-181. DOI: 10.1080/19331681003749030

Williamson, A. (2010). Inside the digital campaign. En The Internet and the 2010 election: Putting the small ' $p$ ' back in politics? Editado por Rachel K. Gibson, Andy Williamson \& Stephen Ward (17-26). Londres: Hansard.

Xenos, M., Vromen, A., y Loader, B. D. (2014). The great equalizer? Patterns of social media use and youth political engagement in three advanced democracies. Information, Communication \& Society, 17(2), 151167. DOI: 10.1080/1369118x2013.871318 